\title{
The influence of modern TV sets and monitors on the parameters defining the quality of electricity and the operation and safety of power grids
}

\author{
Andrzej Lange ${ }^{1, *}$, Marian Pasko ${ }^{2}$ \\ ${ }^{1}$ University of Warmia and Mazury in Olsztyn, Department of Electrical and Power Engineering, Electronics and Automation, EDP \\ Sciences, Editorial Department, 10-736 Olsztyn, ul. Oczapowskiego 11, Poland \\ ${ }^{2}$ Silesian University of Technology, Institute of Electrical Engineering and Computer Science, 44-100 Gliwice, Akademicka 2A, Poland
}

\begin{abstract}
This paper overviews European Commission regulations, directives of the European Parliament and the European Council, Polish laws and regulations as well as standards applicable to power quality, in particular the power factor and the higher harmonic content of currents and voltages in power grids. Power quality parameters for loads such as TV sets and monitors were measured and analyzed. The time waveforms of currents for these loads during standard operation and in stand-by mode were presented. The influence of the evaluated devices on the design and operation of modern power grids was analyzed with special emphasis on electrical safety.
\end{abstract}

\section{Preface}

The article presents the regulations and standards applicable to the quality of electricity, in particular the higher harmonic content of currents and voltages in power grids. Power quality parameters for loads such as TV sets and monitors were measured and discussed. The time waveforms of currents for these loads during standard operation and in stand-by mode were presented. The influence of the evaluated devices on the design and operation of modern power grids was analyzed, with special emphasis on electrical safety and fire protection.

\section{Standards and regulations on power quality and the general structure of power supply circuits in TV sets and monitors}

Selected provisions of the European Union directives, Polish regulations and standards are presented in the References section in points [1] to [8]. These regulations define the minimum requirements for power quality and supply voltage (higher harmonic frequencies), power factor $\cos \varphi$, and power grid requirements, including electrical safety and fire protection.

Modern TV sets and monitors have the following components: a rectifier circuit, a capacitor for smoothing the voltage supplied by the rectifier, and a currentvoltage regulator. The complexity of the power supply system varies in different types of devices, which significantly influences current waveforms.

\section{Measurements and analysis of results}

The quality of electricity drawn by TV sets and monitors was analyzed by testing more than ten randomly selected devices with the use of the HIOKI 3196 power quality analyzer. The analyses involved measurements of current, voltage and power as well as higher current and voltage harmonics drawn by the tested devices.

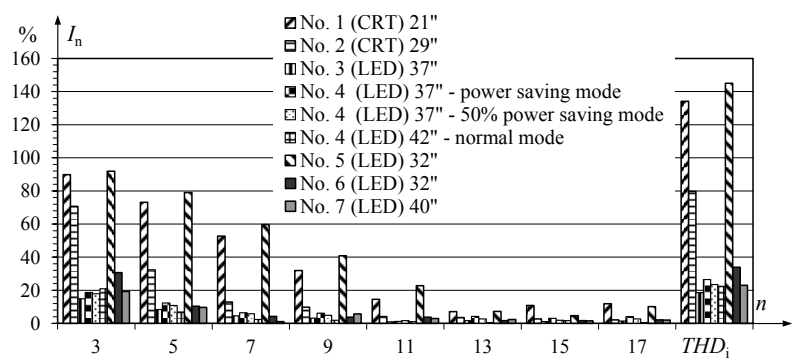

Fig.1. Percentage of higher current harmonics and $T H D_{\mathrm{I}}$ drawn from a low-voltage power grid by TV sets during operation.

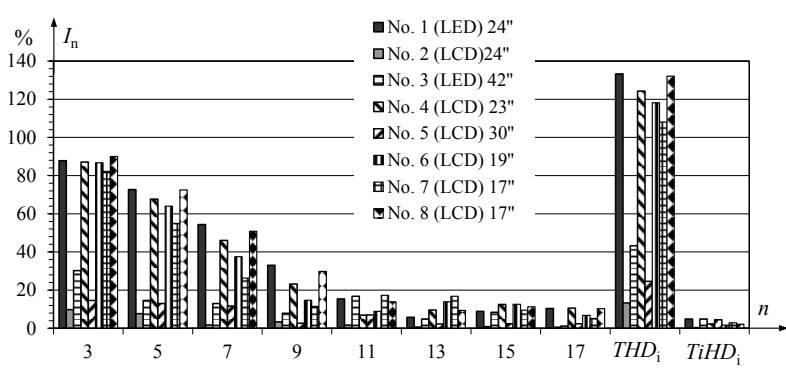

Fig.2. Percentage of higher current harmonics and $T H D_{\mathrm{I}}$ and $T i H D_{\text {I }}$ drawn from a low-voltage power grid by monitors during operation. Part 1. 
Current and voltage waveforms during the operation of the tested devices were also measured. The registered higher current harmonics generated by TV sets to the grid are presented in Figure 1, and the harmonics generated by monitors are presented in Figures 2 and 3 . The phase angle between current and voltage for the first harmonic is presented in Figure 4. Current waveforms during the operation of monitors are presented in Figures $5 \mathrm{a}$ and $5 \mathrm{~b}$.

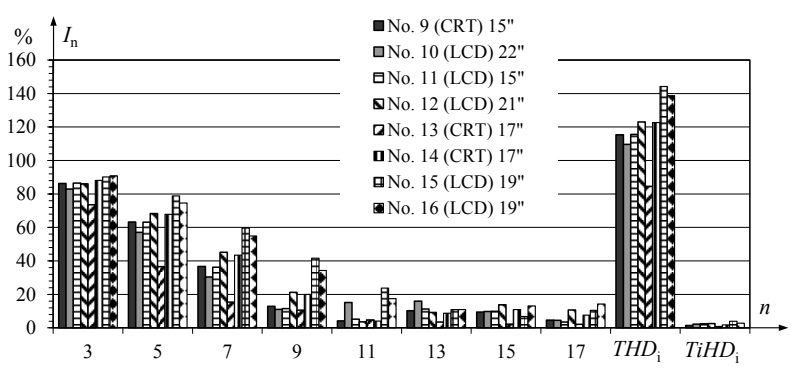

Fig.3. Percentage of higher current harmonics and $T H D_{\mathrm{I}}$ and $T i H D_{\text {I }}$ drawn from a low-voltage power grid by monitors during operation. Part 2.

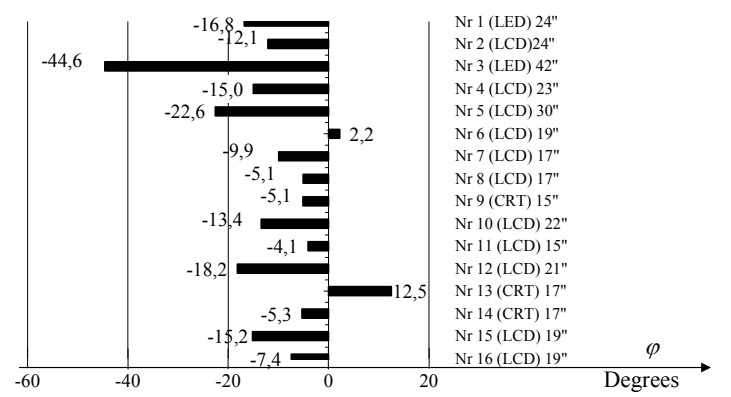

Fig.4. Phase angle between voltage and current for the first harmonic during the operation of the tested monitors. a)

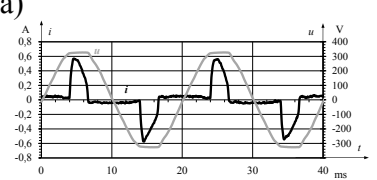

b)

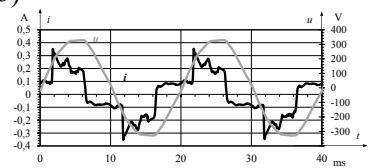

Fig.5. Current waveform during the operation of: a) monitor No. 10 (LCD “22), b) monitor No. 3 (LCD 42”).

\section{Conclusions}

Polish and EU regulations do not specify the maximum higher harmonic frequencies that can be generated by domestic electrical appliances, excluding light sources. The relevant regulations specify only the amount of energy drawn from the grid by different types of appliances. Modern TV sets and monitors (LED) as well as older TV sets (CRT):

- draw deformed current by generating higher current harmonics to the power grid (Fig. 1, 2 and 3),

- some TV sets and monitors are equipped with simple rectifier systems with a capacitor for smoothing the voltage transmitted to internal circuits; therefore, they draw pulsed current (Fig. 5a and 5b) and generate higher values of higher harmonics reaching $T H D_{\text {I }}=140 \%$ (Fig. 1, 2 and 3),

- the power factor can be positive or negative in modern devices, which can complicate passive power compensation, in particular when the contribution of higher harmonics is very high,
- higher harmonics in supply voltage increase with the absorption of higher current harmonics, which can lead to electrical current resonance,

- during normal operation, LED TV sets are capacitive loads and tube TV sets are inductive loads in the power grid,

- in stand-by mode, all of the tested TV sets and monitors are capacitive loads drawing higher harmonics from the power grid,

- very high values of the third harmonic can cause excessive neutral loads because third harmonics are summed up in three-phase systems. Wire gauge standards and protection standards should be modified. Protection should also be used in neutral conductors, i.e. integrated two-way connectors for single-phase circuits and four-way connectors for three-phase circuits,

- residual current circuit breakers may not offer effective protection in devices that draw pulsed current. Residual current circuit breakers are mandatory, but their type is not specified by the relevant regulations. Cheap type AC breakers are most often used, but they do not offer any protection against pulsed current. Modern TV sets and monitors should be provided with type A breakers [29], [30]. Similar solutions are found in other domestic appliances, such as washing machines and refrigerators.

\section{References}

1. Polish Power Law of 25 September 2012. Journal of Laws, item 1059, volume 1

2. Directive 2014/30/EU of the European Parliament and of the Council of 26 February 2014 on the harmonisation of the laws of the Member States relating to electromagnetic compatibility

3. Regulation of the Minister of the Economy of 4 May 2007 on the detailed operating requirements for the electric power system.

4. EN 50160: 1998. Voltage characteristics of electricity supplied by public distribution systems

5. IEEE Std 1459-2010 Standard Definitions for the Measurement of Electric Power Quantities Under Sinusoidal, Nonsinusoidal, Balanced, or Unbalanced Conditions; IEEE, New York, 2010

6. EN 61000-3-2:2014-10: Electromagnetic compatibility (EMC) - Part 3-2: Limits for harmonic current emissions (equipment input current $\leq 16 \mathrm{~A}$ per phase)

7. EN 61008-1:2013-05: Residual current operated circuit-breakers without integral overcurrent protection for households and similar uses (RCCBs) - Part 1: General rules

8. Regulation of the Minister of Infrastructure of 12 April 2002 on the technical requirements for buildings and their location. 